\title{
Can clopidogrel and aspirin lower mortality in patients with acute myocardial infarction?
}

Deepak L Bhatt

Nature Clinical Practice Cardiovascular Medicine (2006) 3: 182-183 [doi: 10.1038/ncpcardio0508]

doi:10.1038/ncpcardio0657

In the April 2006 issue, the second sentence of the Results section was published as "The COMMIT investigators found that the group who received aspirin plus clopidogrel had significantly fewer reinfarctions, strokes and deaths than the group given aspirin and clopidogrel". This should have appeared as "The COMMIT investigators found that the group who received aspirin plus clopidogrel had significantly fewer reinfarctions, strokes and deaths than the group given aspirin and placebo".

\section{Is rimonabant a safe and effective therapy for sustained weight loss and improved cardiometabolic risk factors?}

Serena Tonstad

Nature Clinical Practice Cardiovascular Medicine (2006) 3: 364-365 [doi: 10.1038/ncpcardio0598]

doi:10.1038/ncpcardio0658

In the July 2006 issue, the third paragraph, line 14 of the Commentary was published as "Furthermore, because of loss of randomization some of the effects of rimonabant on lipid levels, insulin and homeostasis model assessments, which were twice that expected as a result of weight loss alone, have also been shown with other drugs. ${ }^{4}$ Statistical analyses alone cannot show that anti-obesity therapy improves a patient's risk factor profile beyond weight loss". This should have appeared as "Furthermore, some of the effects of rimonabant on lipid levels, insulin and homeostasis model assessments, which were twice that expected as a result of weight loss alone, have also been shown with other drugs. ${ }^{4}$ Statistical analyses alone cannot show that antiobesity therapy improves a patient's risk factor profile beyond weight loss because of loss of randomization." 